\title{
Welder's pulmonary hemosiderosis associated with systemic iron overload following exacerbation of acute adult T-cell leukemia/lymphoma
}

\author{
Naoto Imoto, ${ }^{1)}$ Akira Shiraki, ${ }^{2)}$ Katsuya Furukawa, ${ }^{1)}$ Naoyuki Tange, ${ }^{1,4)}$ Atsushi Murase, ${ }^{1,4)}$ \\ Masaya Hayakawa, ${ }^{1)}$ Yosuke Iwata, ${ }^{3)}$ and Hiroshi Kosugi ${ }^{1)}$
}

Herein, we describe a 61-year-old man diagnosed with pulmonary hemosiderosis following chemotherapy for acute adult T-cell leukemia/lymphoma (ATLL). Liver and heart biopsy confirmed hemosiderosis. ATLL progressed, and the patient died from multiorgan damage. Welder's lung may have been involved in hemosiderosis and systemic iron overload. Abnormal iron metabolism or immune reactions may have influenced the clinical course, but these were not validated. Detailed analyses of family medical and lifestyle histories, and genetic examination should be performed in cases of systemic iron overload.

Keywords: pulmonary hemosiderosis, adult T-cell leukemia lymphoma, systemic iron overload, welder's lung

\section{INTRODUCTION}

Pulmonary hemosiderosis is a disease where excess iron is deposited in the lungs as hemosiderin. This can occur as a consequence of occupations like welding, and in most cases, only the lungs are affected. ${ }^{1-5}$ However, systemic iron overload resulting from several causes can induce tissue damage and organ failure. ${ }^{6-8}$ There are hereditary and acquired types of iron overload, and cases associated with repeated red blood cell transfusion are frequently reported. ${ }^{9}$ Iron overload can damage the liver, heart, pancreas, endocrine organs, and central nervous system. However, deposition of hemosiderin in the lungs is rarely reported. Herein, we report a rare case in which pulmonary hemosiderosis was initially diagnosed following chemotherapy for adult T-cell leukemia/ lymphoma (ATLL), but systemic iron overload was identified later.

\section{CASE REPORT}

A 61-year-old man visited our hospital for complete left bundle branch block detected during a medical checkup in 2010. The patient underwent treatment for acute pneumonia 10 years earlier. Regarding occupational history, the patient had performed welding at an ironworks for 40 years. There was no particular family medical history. He smoked 20 cigarettes per day for 30 years, and drank $500 \mathrm{~mL}$ of beer per day for 30 years.

On examination, we observed moderate aortic stenosis, shadows of diffuse lung infiltration in the bilateral lower lung fields (Fig. 1), and erythema in the trunk. The lung shadow suggested interstitial pneumonia, but the patient rejected examination by bronchoscopy. Therefore, we monitored the course of disease. On close examination at the Department of Dermatology, anti-nuclear, anti-centromere, and anti-ribonucleoprotein antibody levels were 1,280-times, 179.5-times, and 26.7-times the normal levels, respectively. Skin biopsy was negative for conditions such as sclerema or collagen disease.

In June 2011, leukocytosis and a 43\% increase in peripheral atypical lymphocytes were observed, and acute ATLL was diagnosed at the Division of Hematology. Moreover, the patient's ferritin level was markedly high $(3,209 \mathrm{ng} / \mathrm{mL})$. On bone marrow examination, hemophagocytosis and macrophage activation were not observed, and no fever or symptoms of adult Still's disease were observed. Therefore, we concluded that ATLL was the cause of hyperferritinemia. Chemotherapy (modified LSG-15 regimen) for ATLL was initiated in the same month, and was completed in January 2012. This resulted in partial remission. During the course of treatment, transfusion with 20 units of red blood cells was performed for myelosuppression due to chemotherapy. Doxorubicin $\left(420 \mathrm{mg} / \mathrm{m}^{2}\right)$ was administered during chemotherapy.

The patient began complaining of shortness of breath in February 2012. On X-ray and computed tomography, there was a marked increase in ground glass opacity (Fig. 2). The patient's body temperature was $36.9^{\circ} \mathrm{C}$, peripheral capillary 
oxygen saturation was $91 \%$ (room air), and no findings suggested recurrence of ATLL, reduction of cardiac function, or infection at the time of visit (Table 1). On bronchoscopic lung biopsy, accumulation of hemosiderin was observed in the alveolar walls and interstitium, and the patient was diagnosed with pulmonary hemosiderosis (Fig. 3a,b).

Welder's lung induced by fume inhalation was considered to be the cause of pulmonary hemosiderosis, resulting from his occupation as a welder. However, marked accumulation of hemosiderin was also observed in the liver cells on biopsy (Fig. 3c-e), demonstrating the presence of systemic iron overload. Steroid pulse treatment at $1 \mathrm{~g}$ /day was performed for 3 days at the beginning of administration, followed by gradual dose reduction and discontinuation. When pulmonary hemosiderosis was diagnosed, administration of 1,000 mg/ day deferasirox was initiated. The respiratory condition improved and the patient was discharged. After 6 months, ground glass opacity was not improved, and no decrease in
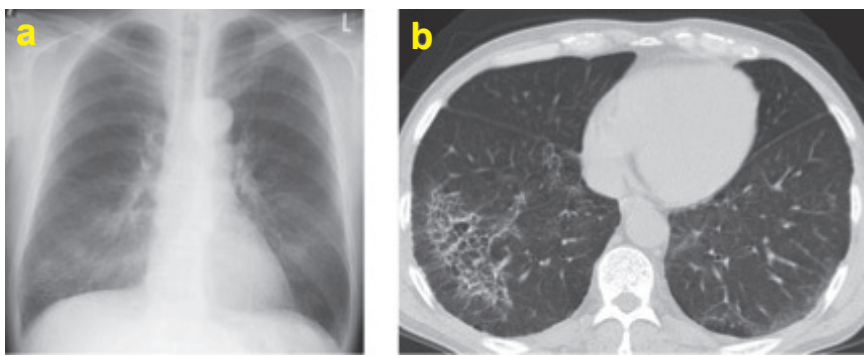

Fig. 1. ( $(\boldsymbol{a}-\boldsymbol{b})$ On plain chest radiography, bilateral alveolar opacity was observed. It was faint, and interstitial pneumonia was not apparent. ferritin was observed. Therefore, administration of the drug was stopped.

ATLL was controlled for a certain period of time, but disease progression was observed in September 2012. An anti$\mathrm{CC}$ chemokine receptor 4 antibody, mogamulizumab, was administered for eight cycles, and partial remission was achieved.

In February 2014, exertional dyspnea developed without an inducer, and a new shadow was observed in the bilateral
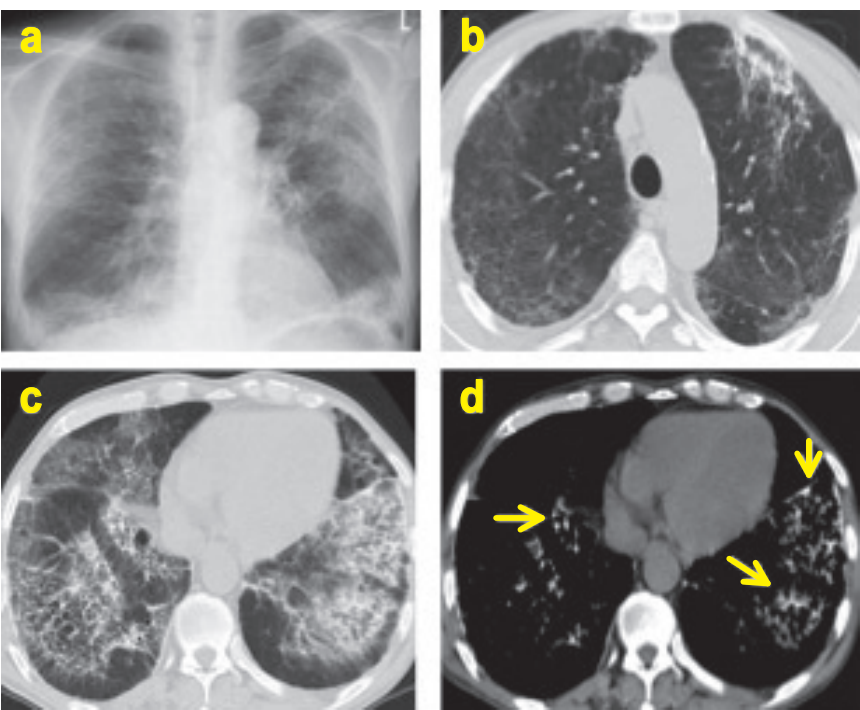

Fig. 2. (a-d) On plain chest radiography, diffuse ground glass opacity (GGO) was observed. (d) With mediastinal window settings, a granular high absorption range was observed in accordance with the area of GGO $(\downarrow)$

Table 1. Laboratory results at the time of examination of pulmonary failure

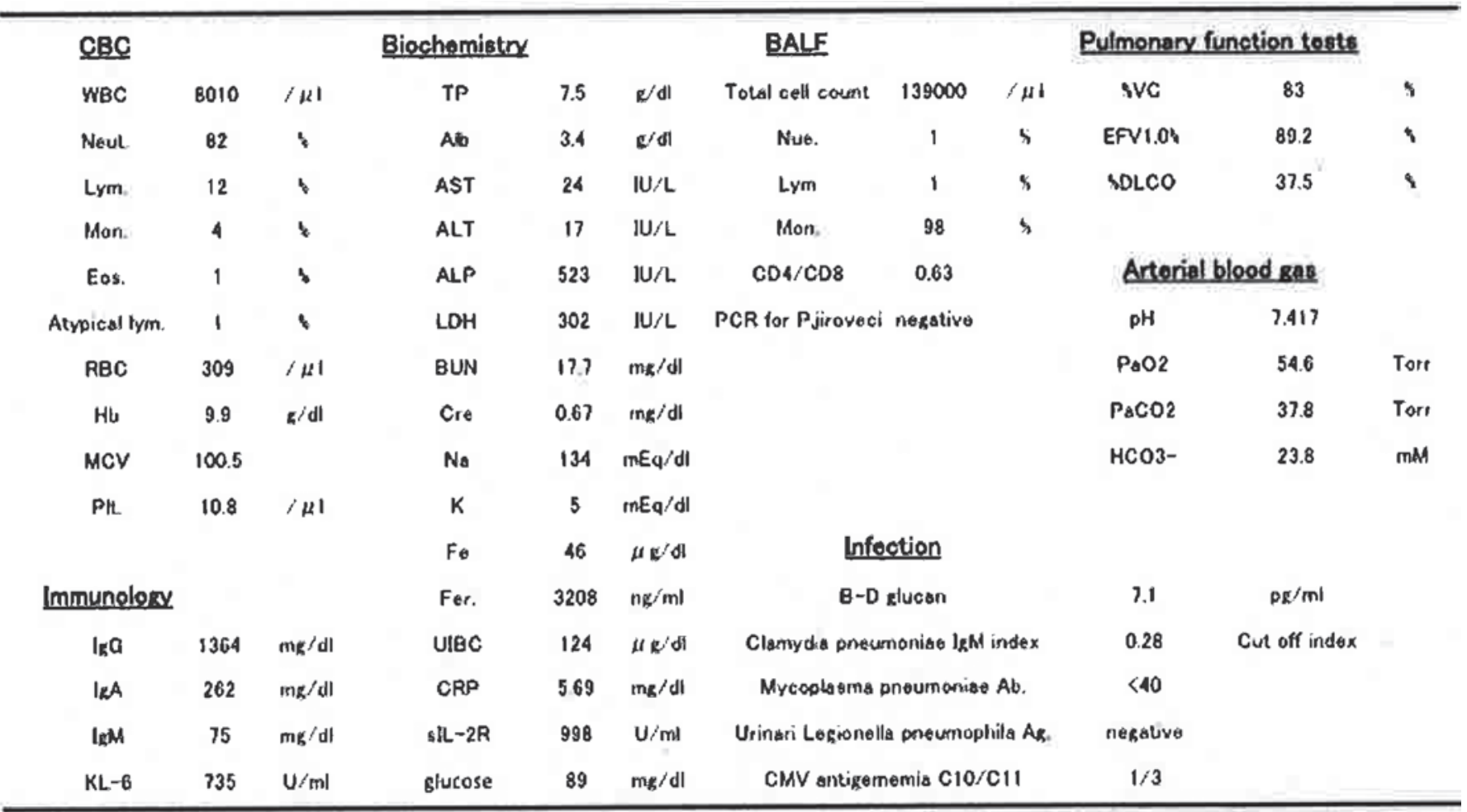


lung. No signs of recurrence of ATLL were noted. The condition was determined to be atypical aggravation of pulmonary hemosiderosis. The levels of brain natriuretic peptide increased to $1,354.7 \mathrm{pg} / \mathrm{mL}$, and the ejection fraction was $27 \%$ on echocardiography, which was considered to be caused by heart failure. Treatment of heart failure improved the respiratory condition. Aortic stenosis and doxorubicininduced myopathy during the treatment of ATLL were considered to be the causes of heart failure, although cardiac hemosiderosis was diagnosed by heart muscle biopsy (Fig. $3 \mathrm{f})$.

In August 2014, progression of ATLL was observed, and
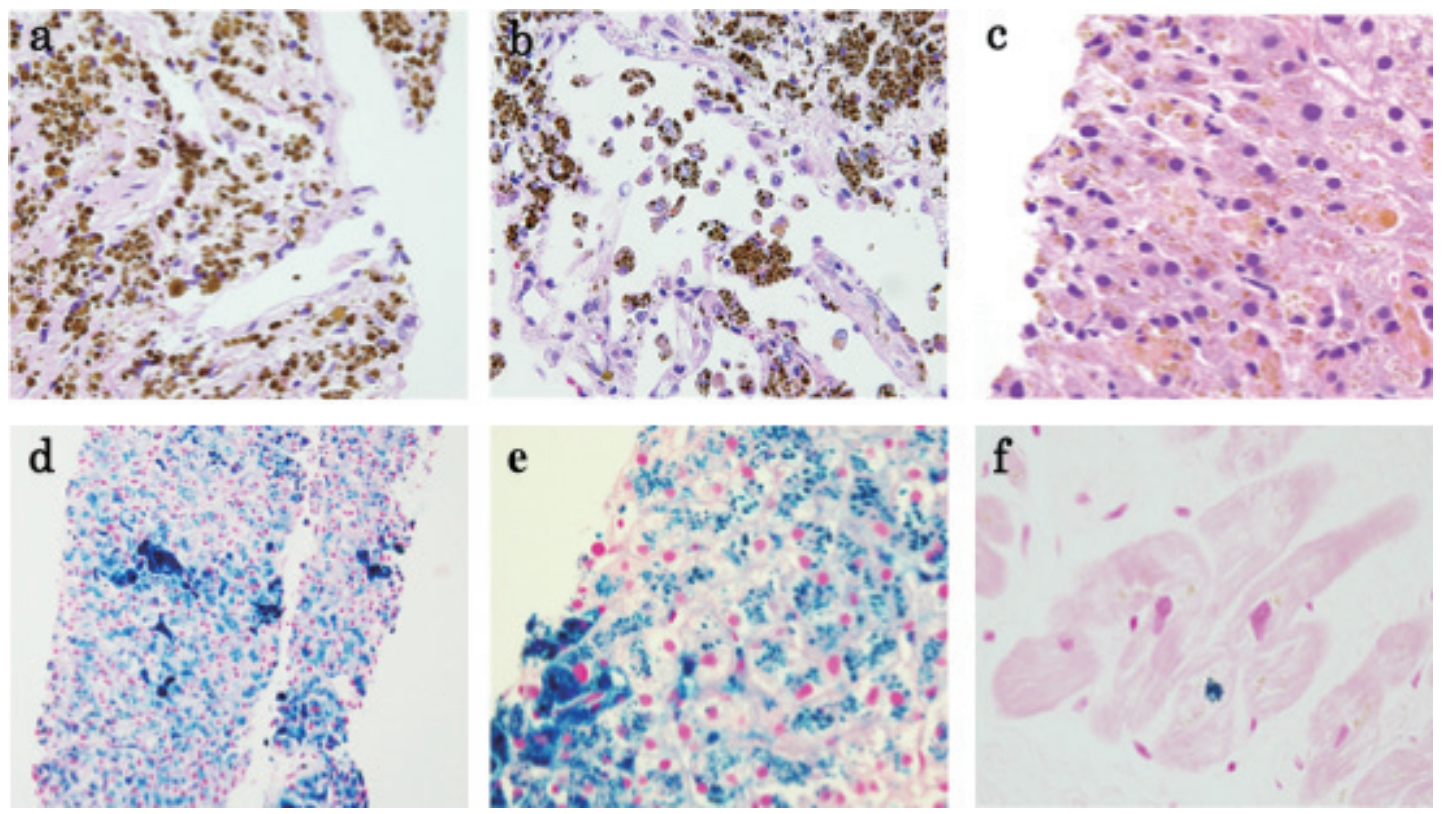

Fig. 3. ( $(\boldsymbol{a}-\boldsymbol{b})$ On lung biopsy, marked accumulation of hemosiderin was observed in alveolar walls and the interstitium. Hemosiderin-containing macrophages were also observed in the alveolar space. $(\boldsymbol{c}-\boldsymbol{e})$ On liver biopsy, accumulation of hemosiderin granules were readily observable in liver cells. Kupffer cells that engulfed hemosiderin were observed in the sinusoid. The granules were strongly positive for Berlin blue staining. $(f)$ On myocardial biopsy, hemosiderin (stained blue with Berlin blue) was observed in small amounts. There was no involvement of ATLL cells in the lung, liver, or heart specimens. ( $\boldsymbol{a}$ : Hematoxylin and Eosin staining $(\mathrm{H} \& \mathrm{E}) \times 200, \boldsymbol{b}$ : $\mathrm{H} \& \mathrm{E} \times 400, c: \mathrm{H} \& \mathrm{E} \times 200, \boldsymbol{d}$ : Berlin blue staining $(\mathrm{BB}) \times 100, \boldsymbol{e}: \mathrm{BB} \times 200, f: \mathrm{BB} \times 200$ )

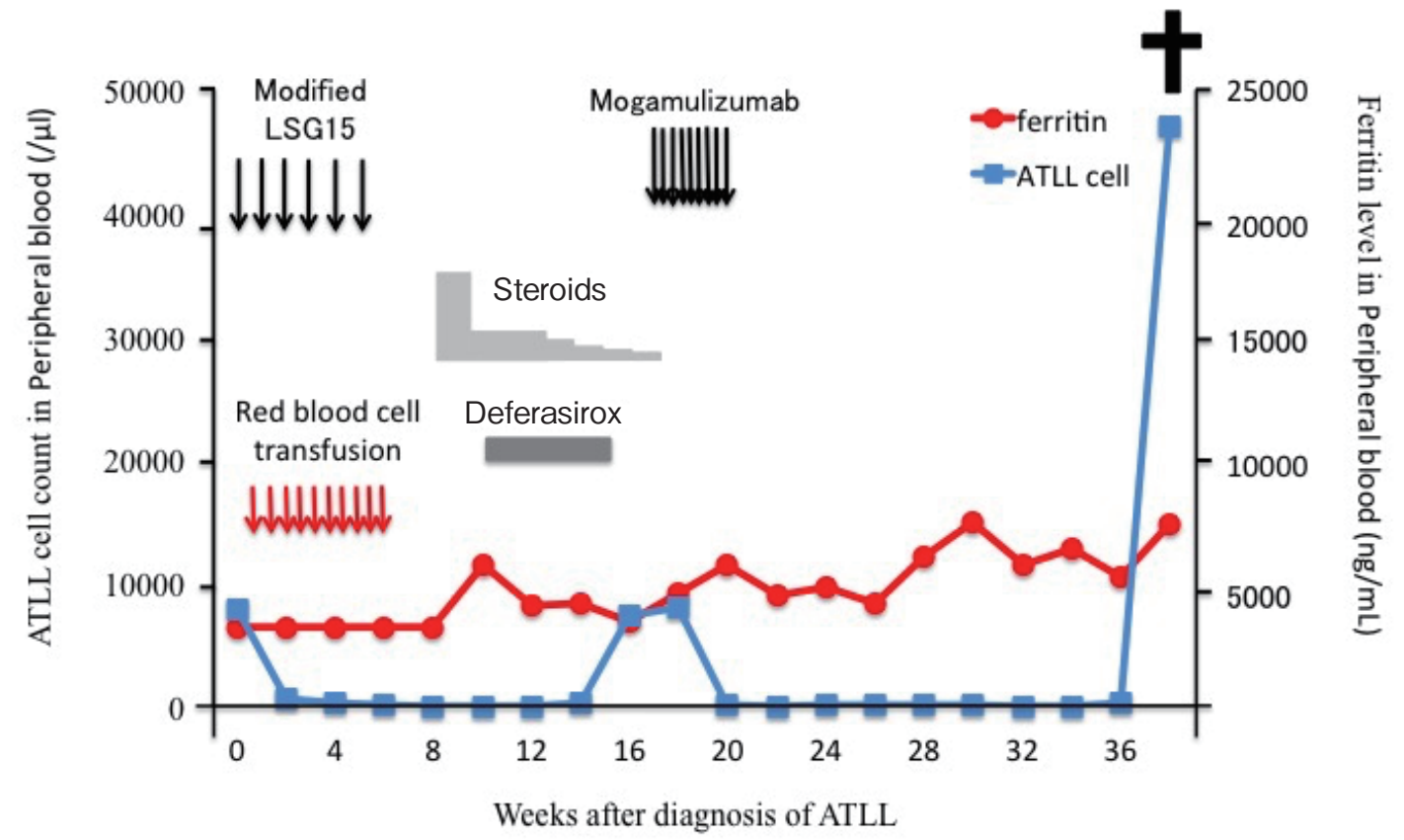

Fig. 4. Clinical course and ferritin levels beginning from the diagnosis of ATLL 
mogamulizumab was readministered. However, the respiratory condition aggravated rapidly on the following day, and the patient died. Pathological autopsy was not performed. Among all pathology specimens from the initial diagnosis, there was no involvement of ATLL cells in the lung, liver, heart, cerebrospinal fluid, or skin. On histology, bone marrow was the only validated site of tumor cell invasion. The clinical course and ferritin levels are shown in Fig. 4.

\section{DISCUSSION}

We identified pulmonary hemosiderosis and systemic iron overload in a patient with ATLL. The simplest and most frequently used method to diagnose iron overload is measurement of serum ferritin. However, increased serum ferritin is caused by several conditions, including inflammatory diseases, tissue damage, hematological malignancy, and immunodeficiency, and it does not accurately assess iron overload. ${ }^{10,11}$ According to the clinical course shown in Fig. 4, the ferritin level did not improve following therapy for ATLL. In addition, no other causes of iron overload were found. Therefore, the increase in ferritin may have primarily been caused by iron overload, which was possibly aggravated by ATLL or chemotherapy. Systemic hemosiderosis may have been present before the onset of ATLL. It may have been advantageous to screen for systemic iron overload using magnetic resonance imaging or biopsy at the time hyperferritinemia was observed, even if acute hematological malignancy was present.

We believe welder's lung caused by fume inhalation was the primary etiology. Welder's lung is caused by the accumulation of the main component of welding fumes, iron oxide, in the lungs, ${ }^{1,2}$ and has been reported to induce diffuse lung disorders. ${ }^{3-5}$ Long-term welding work (40 years) may have explained the development of pulmonary hemosiderosis in the present case. Although welder's lung is usually limited to the lungs and systemic iron overload is rare, there are reports that welder's lung can lead to systemic iron overload. ${ }^{12,13}$ The mechanism remains unknown, and it is assumed that systemic iron absorption via lung macrophages may be present for a long period of time.

However, it is doubtful that welder's lung alone was the primary cause of hemochromatosis in the present case. As noted above, systemic iron overload is rare in patients with welder's lung. Additionally, disease onset in our patient occurred after exposure to welding fumes for $>6$ months, and his basal ferritin levels were consistently elevated, regardless of welding fume exposure. This suggests that systemic iron overload was caused by other factors.

Having received red blood cell transfusion is the most important factor for the differential diagnosis of iron overload. It has been reported that serum ferritin levels exceed $1,000 \mathrm{ng} / \mathrm{mL}$ in approximately $50 \%$ of patients when $\geq 20$ units of blood are transfused, ${ }^{9}$ which can cause iron overload. However, we believe that blood transfusion was a minor cause of iron overload in the present case because infusion of 20 units of red blood cells alone is not sufficient to cause severe iron overload, and the lungs are not the most commonly affected organ. In addition, the patient's ferritin level was markedly high before blood transfusion, and did not change after transfusion.

There are no reports that leukemia, lymphoma, or chemotherapy cause systemic iron overload. Therefore, ATLL may not be related with hemosiderosis. However, we believe there was an immunological association between the deterioration of the respiratory condition or lung shadow, and chemotherapy. This is because respiratory failure occurred soon after the completion of chemotherapy, steroids were effective for treatment, and there was no fume exposure during the deterioration of the respiratory condition. However, the pathogenesis of iron-induced pulmonary disease remains unclear, and there are several reports that demonstrated an immunological mechanism in welder's lung., ${ }^{2,5,14}$

One condition other than blood transfusion that induces iron overload is hereditary hemochromatosis caused by mutations in the HFE gene..$^{15,16}$ Family medical history is important when assessing hereditary hemochromatosis. ${ }^{17}$ There are also several secondary causes, such as alcoholic cirrhosis and diet, ${ }^{18,19}$ indicating that assessment of lifestyle history is important. The family medical and lifestyle histories of our patient were unremarkable. However, we cannot exclude the possibility of the presence of genetic hemochromatosis because molecular genotyping for common mutations was not performed. If there were no genetic causes, an unknown etiology of abnormal iron metabolism may have caused atypical iron overload.

In conclusion, in the present case, the course of pulmonary hemosiderosis and systemic iron overload may have been associated with welder's lung. Despite the lack of definitive evidence, we believe abnormal iron metabolism and immune reactions from chemotherapy may have affected the clinical course of hemosiderosis. When hyperferritinemia is observed, systemic iron overload should always be considered as a differential diagnosis. If it is identified, the cause should be closely investigated, and genetic examination should be performed.

\section{CONFLICTS OF INTEREST}

The authors declare that they have no conflicts of interest.

\section{REFERENCES}

1 Lombardo LJ, Balmes JR: Occupational asthma: a review. Environ Health Perspect 108: 697-704, 2000

2 Fontenot AP, Amicosante M: Metal-induced diffuse lung disease. Semin Respir Crit Care Med 29: 662-669, 2008

3 Kunimasa K, Arita M, Tachibana H, Tsubouchi K, Konishi S, et al.: Chemical pneumonitis and acute lung injury caused by inhalation of nickel fumes. Intern Med 50: 2035-2038, 2011

4 Taniguchi H, Suzuki K, Fujisaka S, Honda R, Abo H, et al.: Diffuse alveolar damage after inhalation of zinc oxide fumes. Nihon Kokyuki Gakkai Zasshi 41: 447-450, 2003

5 Antonini JM, Lewis AB, Roberts JR, Whaley DA: Pulmonary 
effects of welding fumes: review of worker and experimental animal studies. Am J Ind Med 43: 350-360, 2003

6 Andrews NC: Forging a field: the golden age of iron biology. Blood 112: 219-230, 2008

7 Cook JD, Barry WE, Hershko C, Fillet G, Finch CA: Iron kinetics with emphasis on iron overload. Am J Pathol 72: 337-344, 1973

8 Kohgo Y, Ikuta K, Ohtake T, Torimoto Y, Kato J: Body iron metabolism and pathophysiology of iron overload. Int J Hematol 88: 7-15, 2008

9 Takatoku M, Uchiyama T, Okamoto S, Kanakura Y, Sawada K, et al.: Retrospective nationwide survey of Japanese patients with transfusion-dependent MDS and aplastic anemia highlights the negative impact of iron overload on morbidity/mortality. Eur J Haematol 78: 487-494, 2007

10 Wood JC: Use of magnetic resonance imaging to monitor iron overload. Hematol Oncol Clin North Am 28: 747-764, 2014

11 Puliyel M, Sposto R, Berdoukas VA, Hofstra TC, Nord A et al.: Ferritin trends do not predict changes in total body iron in patients with transfusional iron overload. Am J Hematol 89: 391-394, 2014

12 Doherty MJ, Healy M, Richardson SG, Fisher NC: Total body iron overload in welder's siderosis. Occup Environ Med 61: 82-85, 2004
13 Patel RR, Yi ES, Ryu JH: Systemic iron overload associated with Welder's siderosis. Am J Med Sci 337: 57-59, 2009

14 Kelleher P, Pacheco K, Newman LS: Inorganic dust pneumonias: the metal-related parenchymal disorders. Environ Health Perspect 108: 685-696, 2000

15 Feder JN, Gnirke A, Thomas W, Tsuchihashi Z, Ruddy DA, et al.: A novel MHC class I-like gene is mutated in patients with hereditary haemochromatosis Nat Genet 13: 399-408, 1996

16 Olynyk JK, Cullen DJ, Aquilia S, Rossi E, Summerville L, et al.: A population-based study of the clinical expression of the hemochromatosis gene. N Engl J Med 341: 718-724, 1999

17 Acton RT, Barton JC, Passmore LV, Adams PC, McLaren GD, et al:: Accuracy of family history of hemochromatosis or iron overload: the hemochromatosis and iron overload screening study. Clin Gastroenterol Hepatol 6: 934-938, 2008

18 Bonkovsky HL, Lambrecht RW, Shan Y: Iron as a co-morbid factor in nonhemochromatotic liver disease. Alcohol 30: 137144, 2003

19 Pietrangelo A: Iron and the liver. Liver Int 36: 116-123, 2016 